\title{
Markov Reward Approach and Reliability Associated Cost Model for Machine Tools Maintenance-Planning Optimization
}

\author{
Zeng Wenbin a,c, Ilia Frenkel ${ }^{b *}$, Shen Guixianga, Igor Bolvashenkov ${ }^{c}$, Jörg \\ Kammermann $^{c}$, Hans-Georg Herzog ${ }^{c}$, Lev Khvatskin ${ }^{b}$ \\ ${ }^{a}$ School of Mechanical and Aerospace Engineering, Jilin University, China \\ ${ }^{\mathrm{b}}$ Center for Reliability and Risk Management, SCE-Shamoon College of Engineering, Israel \\ ${ }^{\mathrm{c}}$ Institute of Energy Conversion Technology, Technical University of Munich, Munich, Germany \\ *Corresponding author: iliaf@frenkel-online.com
}

(Received November 19, 2018; Accepted April 25, 2019)

\begin{abstract}
This paper proposes a novel Reliability Associated Cost (RAC) model for machine tools throughout its lifetime that considers two different failure consequences, immediate failure and product rejections increase failure. A maintenance strategy of corrective maintenance combined with overhaul utilized to the maintenance activities of machine tools in the current paper. Markov reward approach is developed for computing of the costs incurred by both failure consequences and maintenance activities and system average availability throughout the machine tools life cycle. The Genetic Algorithm is used to find the optimal repair rates layout and overhaul moments that provide a minimal expected cost of system operation and maintenance actions and satisfies the desired availability requirement. A numerical example is presented in order to illustrate the approach and the results show that the proposed technique can significantly cut the RAC for machine tools.
\end{abstract}

Keywords- Markov reward approach, Reliability associated cost model, Machine tools, Optimal maintenance-planning.

\section{Introduction}

Maintenance is an activity, in which repairing is carried out at certain intervals, to extend the useful life of the machine (Gandhi and Ng, 2018). Reason (2000), Sharma et al. (2011) define maintenance as those activities required to keep a facility in "as built" condition and therefore continuing to have its original productive capacity. For the machine tools maintenance also effects the product quality and productivity (Lad et al., 2016). Therefore, for machine tools, an important issue is how to reduce maintenance cost and manage business risks while increasing asset reliability, availability and safety (Atamuradov et al. 2017). Thus, maintenance planning and its cost of machine tools continue, over the years and still, to draw the attention of production management since the unplanned failures decrease the reliability of the machine and also profit and competitiveness of the company.

Shagluf et al. (2018) presents a novel decision support system to allow manufacturers to predict the cost of both calibration strategies and provide them with the knowledge to make the best maintenance strategy decision in terms of minimizing financial cost and maximizing machine tools accuracy. Mourtzis et al. (2016) integrate a condition-based preventive maintenance approach into a machine tools monitoring framework, which acquires data from shop-floor machine tools and analyses them through an information fusion technique to support the condition-based preventive maintenance operations. The proposed approach is developed into a software service, deployed on a Cloud environment. In paper Peysson et al. (2019) proposes advanced services that are required for a fleet-wide proactive maintenance platform-KASEM to centralize data and knowledge on 
International Journal of Mathematical, Engineering and Management Sciences

Vol. 4, No. 4, 824-840, 2019

https://dx.doi.org/10.33889/IJMEMS.2019.4.4-065

twelve machine tools and several Generic Algorithms have been developed to evaluate machine health and generate early detection events to anticipate machine failure. Fakher et al. (2017) combine the planning of production, age-based imperfect maintenance and process inspections in a system composed of multiple machines and products. This level of integration is almost new in the literature; it can help practitioners in realizing quality improvement and cost reduction goals. In Ungureanu et al. (2015) is presented the development and implementation of maintenance policies for $\mathrm{CNC}$ machine tool with considering the evolution of defects, their distribution in time and interruptions frequency, and proposes two new approaches to maintenance strategies. Later Ungureanu et al. (2016) introduce a new method, Consequence of Failure Analysis (CFA), based on technical and economic optimization, and was proposed to obtain a level of required performance of CNC machine tools with minimum investment and maintenance costs. In Wang et al. (2016) establishes a fault prediction model of large-scale machine tool joint surface base on the Genetic Algorithm and Back Propagation (BP) neural network. With the help pf this model, the fault after training can be predicted and the maintenance cost and the downtime of the large-scale machine tool can be optimized. Wan et al. (2018) presents a collaborative maintenance-planning framework to connect different stakeholders and integrate their knowledge into the maintenance and service process of a real CNC machine tool. The research realized that advanced content management systems (CMS) have advantages compared with traditional engineering information systems, especially in managing dynamic and unstructured knowledge. In another study Wan et al. (2015), a Collaborative Maintenance Planning System (CoMPS) was proposed to manage planning maintenance operation sequences, schedules and resource allocation to support decision making in machine tools maintenance process planning. In addition, Xu and Cao (2018) proposes an approach to schedule the maintenance activities of machine tools for improving the energy efficiency of the production process, taking into account productivity, product quality, and energy consumption.

The above-mentioned studies analyze the optimal maintenance schedule for machine tools from various aspects; however, the methods for the optimal repair rates or repair capacity planning for different operational periods of a machine tool in its lifetime have not been comprehensively developed. Therefore, a maintenance strategy for machine tools is corrective maintenance (CM) combined with overhaul $(\mathrm{OH})$ considered in this paper, which means the failures occurred before the set overhaul time are fixed through corrective maintenance. Moreover, the repair effect of $\mathrm{CM}$ and $\mathrm{OH}$ regarded as minimum and imperfect maintenance, respectively.

Thus, a comprehensive technique for optimal planning the repair rates and determining the optimal overhaul executed moments to satisfy the demanded availability requirements at the minimal Reliability Associated Cost (RAC) presented in the current paper. The objective is to determine series of repair rates or repair capacity in different operational stages and the number of overhauls as well as corresponding moments, which can provide a minimal system life cycle cost subject to availability constraints. Moreover, the impact of product quality detection process on machine tools states is considered in the current paper to demonstrate the RAC caused during the lifetime of machine tools more realistic. The Markov reward approach is developed for evaluating the availability and expected Reliability Associated Cost of machine tools for each specific time interval. The Genetic Algorithm (GA) is used to solve the combinatorial optimization problem.

\section{Problem Formulation and Description of System Model}

The Reliability Associated Cost (RAC), introduced by Lisnianski et al. (2008), is defined as the total cost incurred by the user in operations and maintenance of the system during its life cycle. Therefore, 
International Journal of Mathematical, Engineering and Management Sciences

Vol. 4, No. 4, 824-840, 2019

https://dx.doi.org/10.33889/IJMEMS.2019.4.4-065

$R A C=C_{o p}+C_{r}+C_{p}$

where $C_{o p}$ is the operation cost of a system accumulated during the system lifetime; $C_{r}$ is the repair cost incurred by the user in repair and maintaining the system during its lifetime; $C_{p}$ is a penalty cost, accumulated during the system life cycle, which was paid when the system failed.

The Reliability Associated Cost is used for many systems, like an air conditioning system for a surgical room (Lisnianski et al., 2007), the traction electric motor for an icebreaking ship (Bolvashenkov et al. 2018) and many others for estimation the total cost of the system during its life cycle.

However, the failures of machine tools may incur additional cost in terms of the product performance. Since there may also be a diversity of opinion between machine tool users and manufacturers as to what exactly constitutes a degraded performance or failure. In Lad et al. (2016), failures of machine tools are defined in terms of failure consequences. Whenever the following consequences occur, machine tools were regarded as failed. The Failure Consequence may be defined as follows:

- Failure Consequence 1 (FC1): failure is detected immediately and the machine has to be stopped.

- Failure Consequence 2 (FC2): machine continues to operate, but produces more rejections than the normal rejection quantity threshold.

The RAC incurred by FC1 is obviously in accordance with formula (1). However, in many practical cases, the users detect FC2 after a time lag, during which the machine tool runs at a reduced performance level. Therefore, the RAC incurred by FC2 should also include the cost caused by the increase amount of rejections, designated as $C_{I R}$. Figure 1 depicts these failure consequences on a time-performance curve. It clearly indicates the relationship of machine tool failures with corresponding RAC during its lifetime.

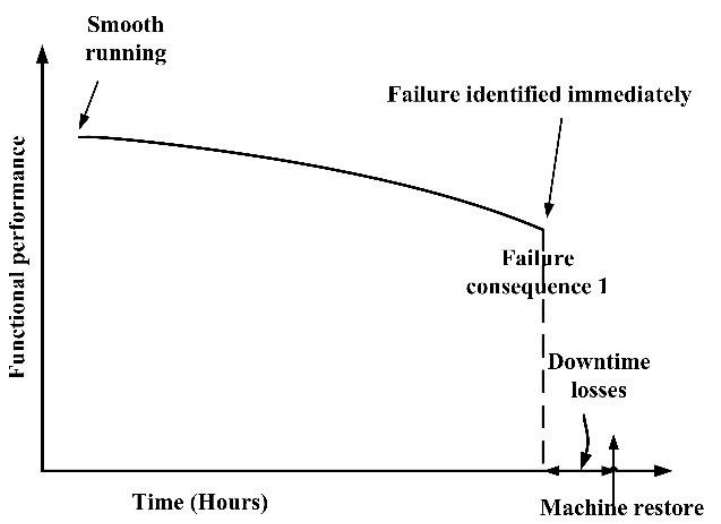

Machine failure due to $\mathrm{FC} 1$

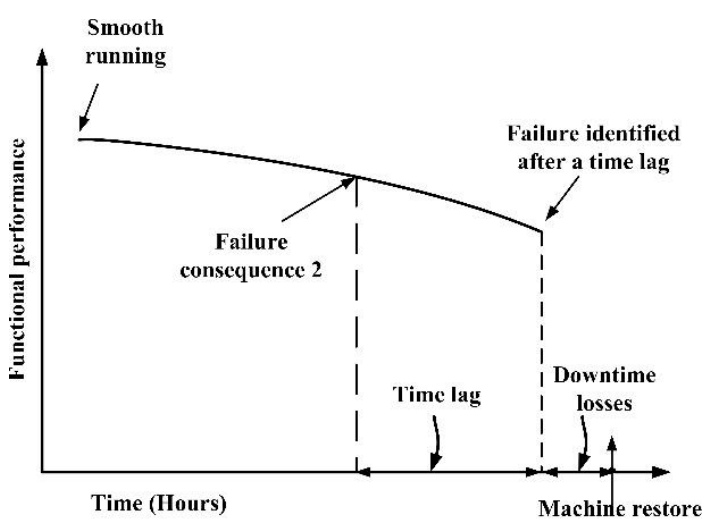

Machine failure due to $\mathrm{FC} 2$

Figure.1 Machine tool failure on time-performance curve 
International Journal of Mathematical, Engineering and Management Sciences

Vol. 4, No. 4, 824-840, 2019

https://dx.doi.org/10.33889/IJMEMS.2019.4.4-065

The maintenance strategy for machine tools is corrective maintenance $(\mathrm{CM})$ combined with overhaul $(\mathrm{OH})$ in this paper, which means the failures occurred before the set overhaul time are fixed through corrective maintenance. Thus, the overhaul cost (designated as $C_{O H}$ ) of a machine tool should also be included in the total RAC during its lifetime. Therefore, the Reliability Associated Cost of machine tools (designated as $R A C_{M T}$ ) that utilize maintenance strategy contains corrective maintenance and overhaul may be presented as follows:

$R A C_{M T}=C_{C M}+C_{O H}=C_{o p}+C_{r}+C_{p}+C_{I R}+C_{O H}$

where $C_{C M}$ is the corrective maintenance cost and $C_{O H}$ is the overhaul cost, $C_{I R}$ is the cost caused by the increased rejections, $C_{o p}, C_{r}$ and $C_{p}$ meaning as mentioned above.

Note. It is supposed that the repair effect of the $\mathrm{CM}$ and $\mathrm{OH}$ is minimal repair and imperfect repair, respectively, which means the repair degree of $\mathrm{CM}$ and $\mathrm{OH}$ is zero and a random value between zero and 1, respectively. The general model proposed by Kijima (1989) can be used to describe the behavior of overhaul as follows:

Assuming the failure rate of a system is $\lambda(t)$ and the moments of overhaul during the system's life cycle are $t_{1}, t_{2}, \ldots, t_{k}$. Therefore,

For $t \in\left[0, t_{1}\right)$

$\lambda_{1}(t)=\lambda(t)$

and the repair degree of the overhaul at the moment $t_{1}$ is $R D_{1}$, then the virtual age $V_{1}$ in $t_{1}$ is,

$V_{1}=R D_{1} \cdot t_{1}$

For $t \in\left[t_{1}, t_{2}\right)$

$\lambda_{2}(t)=\lambda\left(t-V_{1}\right)$

Then the description of the general model shown as follows:

For $t \in\left[t_{k}, t_{k+1}\right)$

$\left\{\begin{array}{c}\lambda_{k+1}(t)=\lambda\left(t-V_{k}\right) \\ V_{k}=R D_{k} \cdot\left(V_{k-1}+t_{k}-t_{k-1}\right)\end{array}\right.$

The initial conditions are: $t_{0}=0, V_{0}=0$.

Let $T$ be the machine tools lifetime and divide into $M$ equal intervals. During each interval the system may be in acceptable state (fully working) or in unacceptable ones (FC1 or FC2). It is assume that FC1 and FC2 are independent and will not occur at the same time. After any failure, a corresponding repair action performed and the system returns to the previously acceptable state. Moreover, the overhaul executed when the increment of RAC of an interval is greater than the overhaul cost. Figure 2 depicts the maintenance activities that performed during the machine tools lifetime. 
International Journal of Mathematical, Engineering and Management Sciences

Vol. 4, No. 4, 824-840, 2019

https://dx.doi.org/10.33889/IJMEMS.2019.4.4-065

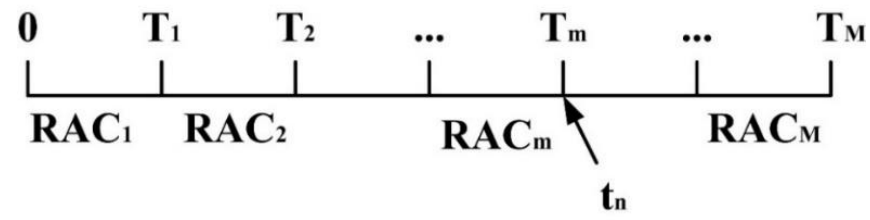

Figure 2. Machine tools maintenance activities

As shown in Figure 2, the overhaul executed at the moment $t_{n}, n=1,2, \cdots, k$ when the following formula is satisfied, which means the RAC incurred in the $(m+1)$ th interval exceeding the overhaul cost.

$R A C_{m+1}-R A C_{m} \geq C_{n}^{O H}$

where $C_{n}^{O H}$ is the cost of $n$ th, $n=1,2, \cdots, k$ overhaul, $R A C_{m}$ is the reliability associated cost incurred until the end of interval $m, m=1,2, \cdots, M$.

Repair rate is the embodiment of the machine tool repair team servicing capacity, which defines some important repair parameters that determine a service level and corresponding costs. The main parameters are mean response time and mean repair time. For simplification only one parameter, mean repair time, $T_{r}^{m}$, where $m(m=1,2, \cdots, M)$ is a time interval of the machine tool lifetime.

The repair cost depends on repair time and is ranged between the most expensive, where repair should be completed during the time $T_{r}^{\min }$ after the failure occurrence and lowest cost, where the repair should be completed during the time $T_{r}^{\max }$ after the failure occurrence. Thus, $T_{r}^{\max } \leq T_{r}^{m} \leq$ $T_{r}^{\mathrm{min}}$ and repair cost corresponding to repair time $T_{r}^{m}$ is designated as $C_{r}^{m}$ for emphasizing its dependence on machine tool aging characteristics. Therefore, the machine tool expected cost of each interval also depends on repair capacity and calculated as follows:

$E\left[R A C_{M T}^{m}\right]=E\left[C_{o p}\right]+E\left[C_{r}^{m}\right]+E\left[C_{p}\right]+E\left[C_{I R}\right]$

Thus, the problem proposed in this paper is to find the optimal maintenance plan for the machine tools that contains the optimal repair rates layout for each interval and the best overhaul moments that can minimize the total expected cost and satisfy the machine tool availability $A(t) \geq A_{0}(t)$, where $A_{0}(t)$ is the minimal machine tool demanded availability.

Therefore, mathematically the problem expressed as follows:

Determine

$\operatorname{MIN}_{\mu^{m}}\left\{\sum_{m=1}^{M} E\left[R A C_{M T}^{m}\right]+E\left[C_{O H}\right]\right\}$

Subject to availability constraint

$A(t) \geq A_{0}(t)$

The mathematical expression of this problem indicates new Reliability Associated Cost model of machine tools that consider the impacts of product quality detection and overhaul on machine 
International Journal of Mathematical, Engineering and Management Sciences

Vol. 4, No. 4, 824-840, 2019

https://dx.doi.org/10.33889/IJMEMS.2019.4.4-065

availability. Therefore, this paper proposes a novel development procedure that based on the new Reliability Associated Cost model to formulate the optimal machine tools maintenance plan. The flow chart is shown in Figure 3.

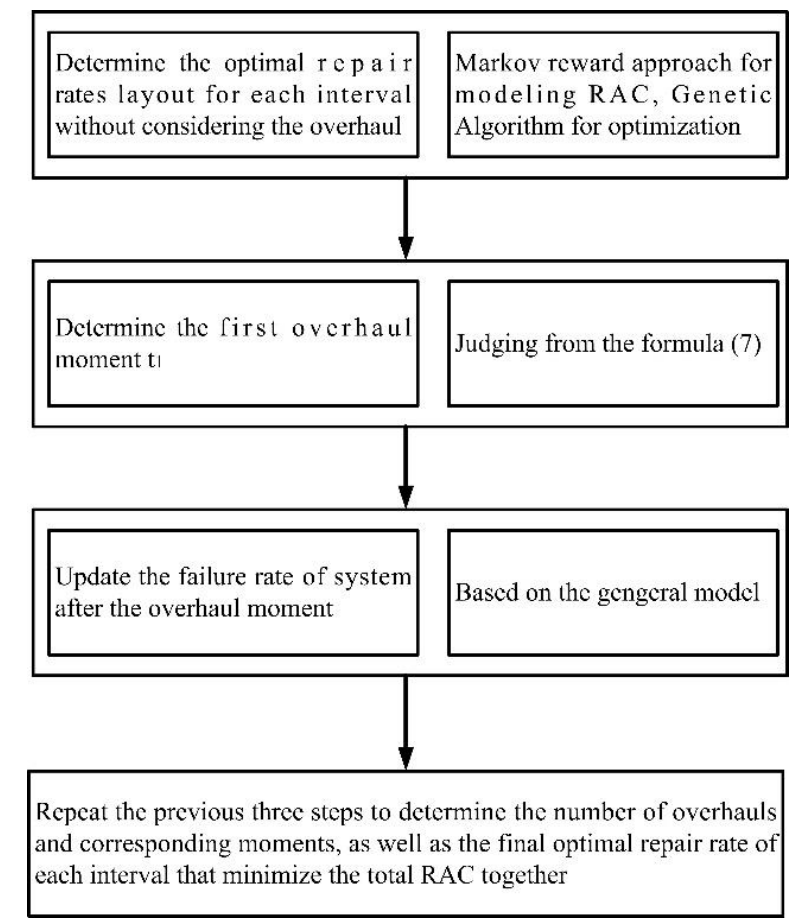

Figure 3. Machine tools maintenance planning process

Based on Lad et al. (2016) and previous research by the authors (Shen et al. 2017), the failure rate of machine tool is assumed to be subject to a two-parameter Weibull distribution.

\section{Markov Reward Approach for Availability and Total Reliability Associated Cost Computation}

A Markov reward approach was firstly introduced by Howard (1960) and applied to the multi-state system (MSS) reliability analysis by Lisnianski and Levitin (2003). A system that can have a finite number of performance rates or distinctive levels of efficiency regarded as a MSS. Detail description of Markov Reward models for Reliability Associated Cost of a MSS presented by Lisnianski (2009), and Lisnianski et al. (2010).

It is assumed that the Markov model for the system has $K$ states that may be represented by a state space diagram as well as transitions between states. Intensities $a_{i j}, i, j=1,2, \cdots, K$ of transitions from state $i$ to state $j$ are defined by corresponding failure and repair rates. For a machine tool, its failure rate $\lambda(t)$ obeys Weibull distribution. In the case of minimal repair, the intensities of transitions from state $i$ to state $j$ corresponding to failures are dependent on time. In addition, in the case of overhaul, the failure rate $\lambda(t)$ calculated through the general model and also dependent on time. 
International Journal of Mathematical, Engineering and Management Sciences

Vol. 4, No. 4, 824-840, 2019

https://dx.doi.org/10.33889/IJMEMS.2019.4.4-065

It is assumed that the system is in any state $i$ during any time unit some payment $r_{i i}$ will be made. It is also assumed that if there is a transition from state $i$ to state $j$ the amount $r_{i j}$ will be paid. The amounts $r_{i i}$ and $r_{i j}$ are called rewards and can be presented as matrix of rewards $\boldsymbol{r}$. The objective is to compute the total expected reward accumulated from $t=0$, when the system begins its evolution in the state space, up to the time $t=T$ under specified initial conditions.

Let $V_{i}(t)$ be the total expected reward accumulated up to time $t$ at state $i$. According to Howard (1960), the following system of differential equations must be solved in order to find this total expected reward:

$\frac{d V_{i}(t)}{d t}=r_{i i}+\sum_{\substack{j=1 \\ j \neq i}}^{K} a_{i j} r_{i j}+\sum_{j=1}^{K} a_{i j} V_{j}(t), i, j=1,2, \cdots, K$

The system (6) should be solved under initial conditions: $V_{i}(0)=0, i=1,2, \cdots, K$.

The expected reward can be found from differential equations (11), by substitution of formulae for failure rates $\lambda(t)$ and repair rate $\mu$ instead of corresponding $a_{i j}$ values.

\subsection{Machine Tool Availability Calculation}

For an availability computation, we partition the set of states $g$, into $g_{0}$, the set of operational or acceptable system states, and $\mathrm{g}_{\mathrm{f}}$, the set of failed or unacceptable states. The system states acceptability depends on the relation between the MSS output performance (designed as $G(t)$, Lisnianski and Frenkel 2009) and the desired level of this performance-demand, which is determined outside the system. In general case demand $W(t)$ is also a random process that can take discrete values from the set $\mathrm{w}=\left\{w_{1}, \ldots, w_{N}\right\}$. The desired relation between the system performance and the demand at any time instant $t$ can be expressed by the acceptability function $\boldsymbol{\Phi}(G(t), W(t))$. The acceptable system states correspond to $\boldsymbol{\Phi}(G(t), W(t))>0$ and the unacceptable states correspond to $\boldsymbol{\Phi}(G(t), W(t))<0$. In many practical cases, the MSS performance should be equal to or exceed the demand. Therefore, in such cases the criterion of state acceptability can be expressed as:

$\boldsymbol{\Phi}(G(t), W(t))=G(t)-W(t) \geq 0$

Here without loss of generality, we assume that required demand level is constant and all system states with performance greater than or equal to $w$ corresponds to the set of acceptable states and all system states with performance lower than $w$ correspond to the set of unacceptable states.

Then the instantaneous availability $A(t)$ of MSS is the probability that the MSS at instant $t>0$ is in one of acceptable states:

$A(t)=\sum_{G(t) \geq W(t)} P_{i}(t)$

Where $P_{i}(t)$ is the probability that at instant $t$ the system is in state $i$.

For a machine tool, an average availability is often used. The average availability is defined in (Lisnianski and Frenkel 2009) as a mean fraction of time when the system resides in the set of acceptable states during the time interval $[0, T]$ : 
International Journal of Mathematical, Engineering and Management Sciences

Vol. 4, No. 4, 824-840, 2019

https://dx.doi.org/10.33889/IJMEMS.2019.4.4-065

$\bar{A}(T)=\frac{1}{T} \int_{0}^{T} A(t) d t$

To assess $\bar{A}(T)$ for machine tool, the rewards in matrix $\boldsymbol{r}$ can be determined in the following manner.

- The rewards associated with all acceptable states should be defined as 1 .

- The rewards associated with all unacceptable states should be zeroed as well as the rewards associated with all transitions.

The total expected reward $V_{i}(T)$ accumulated during interval $[0, T]$ defines a time that machine tool will be in the set of acceptable states in the case where state $i$ is the initial state. This reward should be found as a solution of the system (11). After solving the system (11) and finding $V_{i}(T)$, the machine tool average availability can be obtained for every $i=1,2, \cdots, K$ :

$\overline{A_{i}}(t)=V_{i}(T) / T$

\subsection{Reliability Associated Cost}

The total RAC of a machine tool during its lifetime should contain the normal cost (as shown in (1) and designated as $C_{1}$ ) and the additional cost (designated as $C_{2}$ ) that incurred by the increased rejections.

\subsubsection{Calculation of $\boldsymbol{C}_{\mathbf{1}}$}

To calculate $C_{1}$, the $R A C_{M T}^{m}, m=1,2, \cdots, M$ that for each interval of a machine tool should be obtained, the required rewards in matrix $\boldsymbol{r}$ can be found in the following manner.

- The rewards associated with all acceptable states should be defined as $C_{o p}$.

- The rewards associated with all unacceptable states that caused by FC1 should be defined as $C_{p}$.

- The rewards associated with all unacceptable states that caused by FC2 should be defined as $C_{p}$.

- The rewards associated with transitions, defined repairs and maintenance $C_{r}^{m}$.

The total expected reward $V_{i}(T)$ accumulated during interval $[0, T]$ defines the RAC as the normal cost incurred by the user in operations and maintenance of the system during its lifetime. The reward for a machine tool incurred in the lifetime should be found through iterative calculation of the system (6). Proceed as follows:

(i) Divide machine tool lifetime into $M$ equal intervals and length of each interval is:

$$
\tau=T / M
$$

(ii) Using system (11) to calculate the accumulated reward of machine tool in first interval, $[0, \tau]$, under the initial conditions: $V_{i}^{1}(0)=0, i=1,2, \cdots, K$. Therefore, the RAC can be obtained for every $i=1,2, \cdots, K$ :

$$
R A C_{M T}^{1}=V_{1}^{1}(\tau)
$$


International Journal of Mathematical, Engineering and Management Sciences

Vol. 4, No. 4, 824-840, 2019

https://dx.doi.org/10.33889/IJMEMS.2019.4.4-065

(iii) The accumulated reward of machine tool in second interval, $[\tau, 2 \tau]$, can be calculated by the system (11) under initial conditions: $V_{i}^{2}(\tau)=V_{i}^{1}(\tau), i=1,2, \cdots, K$. Therefore,

$$
R A C_{M T}^{2}=V_{1}^{2}(2 \tau) \text {. }
$$

(iv) Iteratively, the cumulative reward of machine tool in $m$ th interval, $[(m-1) \tau, m \tau]$, is obtained by solving the system (11) under initial conditions: $V_{i}^{m}((m-1) \tau)=$ $V_{i}^{m-1}((m-1) \tau), i=1,2, \cdots, K ; m=1,2, \cdots, M$. Therefore,

$$
R A C_{M T}^{m}=V_{1}^{m}(m \tau) \text {. }
$$

(v) Thus, the normal RAC of a machine tool during interval $[0, T]$ can be obtained for every $i=1,2, \cdots, K$ :

$$
C_{1}=V_{1}^{M}(T)
$$

\subsubsection{Calculation of $\boldsymbol{C}_{2}$}

Furthermore, the machine continues to operate but produces more rejections in the FC2, which incur additional cost (designated as $C_{2}$ ) that includes the additional rejections cost and operation cost can be computed through the system of differential equations (11) for calculating the mean number of FC2 (designated as $N_{F C 2}$ ). The rewards in matrix $\boldsymbol{r}_{N}$ can be determined in the following manner.

- The rewards associated with all transitions to FC2 should be defined as 1 .

- The other rewards in the matrix should be zeroed.

Thus, the total expected reward accumulated during each interval defines the mean number of FC2 is as follows:

$N_{F C 2}^{m}=\left\lceil V_{N 1}^{m}(t)\right\rceil$

where $\left\lceil V_{N 1}^{m}(t)\right\rceil$ indicates the smallest integer larger than $V_{N 1}^{m}(t)$.

Therefore, the additional cost incurred by FC2 during the machine tool lifetime obtained as follow:

$C_{2}=\left(C_{I R}+\left(\frac{1}{f}\right) \cdot C_{o p}\right) \cdot \sum_{m=1}^{M} N_{F C 2}^{m}$

where $f$ is the product quality detection frequency, $C_{I R}$ is the average additional rejections cost.

Therefore, the expected total Reliability Associated Cost of the machine tool during its lifetime adjusted as follows:

$E\left[R A C_{M T}\right]=\sum_{m=1}^{M} E\left[R A C_{M T}^{m}\right]+E\left[C_{O H}\right]=E\left[C_{1}\right]+E\left[C_{2}\right]+E\left[C_{O H}\right]$

\section{Optimization Technique Using Genetic Algorithms}

The genetic algorithms are the most popular heuristic algorithms for solving different kinds of reliability optimization problems. The implementations of GAs in reliability engineering have been applied in the structure optimization and maintenance optimization. 
International Journal of Mathematical, Engineering and Management Sciences

Vol. 4, No. 4, 824-840, 2019

https://dx.doi.org/10.33889/IJMEMS.2019.4.4-065

\subsection{Solution Representation}

To implement GA to a specific problem one has to define the solution representation. A numerical string with length $M$ is used to encode a solution for the optimal repair rates arrangement of a machine tool. A typical chromosome representing repair rates layout is formed as shown in Figure 4.

\begin{tabular}{|c|c|c|c|c|c|}
\multicolumn{1}{|c|}{ Interval 1 } & Interval 2 & $\cdots$ & Interval $m$ & $\cdots$ & Interval $M$ \\
\hline$\mu^{1}$ & $\mu^{2}$ & $\cdots$ & $\mu^{m}$ & $\cdots$ & $\mu^{M}$ \\
\hline \multicolumn{7}{c}{ repair rates layout }
\end{tabular}

Figure 4. GA chromosome structure

The sequence of $M$ numerical items represents the repair rates layout for the whole lifetime of a machine tool. The $M$ items is generated randomly and should be positive numbers. To apply the GA search for the solution with minimal system total expected cost under availability constraint $A(t) \geq A_{0}(t)$, the solution fitness function is follows:

$F=q \cdot \int_{0}^{T} \max \left\{0, A(t)-A_{0}(t)\right\} d t+\sum_{m=1}^{M} E\left[R A C_{M T}^{m}\right]$

where $q$ is a sufficiently large penalty coefficient.

\subsection{Procedure of GA}

The procedure to solve the optimization problem of repair capacity layout of a machine tool using the proposed GA consists of the following steps:

(i) Generate an initial population of solutions randomly;

(ii) Decode each solution and evaluate their fitness;

(iii) Select two solutions randomly and produce pairs of new solutions for the next generations using a crossover procedure. The one-point or two-point crossover method has been used to obtain the feasibility of solutions;

(iv) Allow the offspring to mutate with probability. Mutation results in slight changes in the spring structure and maintains diversity of solutions. This procedure avoids premature convergence to a local optimum and facilities jumps in the solution space;

(v) Apply a selection procedure that compares the new offspring with the worst solutions in the population; and selects the best one. The best solution joins the population and the worst one is discarded;

(vi) Repeat the steps 2-5, until stopping criterion is satisfied. The stopping criterion of the GA can be the fixed number of genetic cycles or a number of genetic cycles without improving the solution performance or both. The convergence criterion in the proposed GA is set as satisfying both a minimal number of genetic cycles and a number of genetic cycles without improving the solution performance;

(vii) Choose the best solution from the population and evaluate its cost. 
International Journal of Mathematical, Engineering and Management Sciences

Vol. 4, No. 4, 824-840, 2019

https://dx.doi.org/10.33889/IJMEMS.2019.4.4-065

\section{Illustrative Example}

To illustrate the application of the present method, consider a single machine whose failure is assumed to follow a two-parameter Weibull distribution with $\alpha=1000 \mathrm{hrs}$ and $\beta=2.2$ as the characteristic life and shape parameter respectively. The machine considered here is expected to operate for two shifts of eight hours each for one day and 300 days a year. The machine tool life period $T=10$ years. Production rate is $20 \mathrm{jobs} / \mathrm{hr}$, the normal rejection rate is 0.05 and quality testing at the end of each shift. Let the probability that it leads to FC1 and FC2 be $P_{F C 1}$ and $P_{F C 2}$ respectively, and in this case $P_{F C 1}=0.7, P_{F C 2}=0.3$. Corresponding system's state-space diagram (Figure 5) has 3 different performance levels: level of perfect functioning (no failure consequence happens), level with failure consequence 1 (immediate failure), and level with failure consequence 2 (the rejection rate increase). After the two failures occur, the machine adopts corrective maintenance and considers that the repair rate is equal in both cases. Moreover, in this case, the overhaul cost of the machine tool set as a constant value $C_{O H}=16000 €$ and the repair degree for each overhaul is $R D=0.8$.

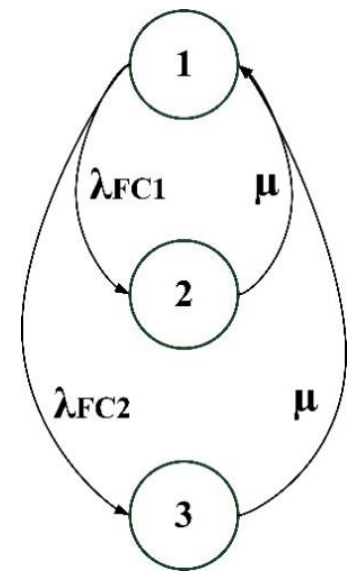

Figure 5. State-space diagram of the machine tool

According to the state space diagram in Figure 5 the following transition intensity matrix $\boldsymbol{a}$ can be as follows:

$\boldsymbol{a}=\left[\begin{array}{ccc}-\left(\lambda_{F C 1}+\lambda_{F C 2}\right) & \lambda_{F C 1} & \lambda_{F C 2} \\ \mu & -\mu & 0 \\ \mu & 0 & -\mu\end{array}\right]$

The failure rate of each failure consequence decided as follows:

$\left\{\lambda_{F C 1}=P_{F C 1} \cdot \lambda(t)\right.$

$\left\{\lambda_{F C 2}=P_{F C 1} \cdot \lambda(t)\right.$

where $\lambda(t)$ is the failure rate of the machine tool.

In order to find the average availability for the machine tool we should present the reward matrix 
International Journal of Mathematical, Engineering and Management Sciences

Vol. 4, No. 4, 824-840, 2019

https://dx.doi.org/10.33889/IJMEMS.2019.4.4-065

$\boldsymbol{r}$ in the following form:

$\boldsymbol{r}=\left[\begin{array}{lll}1 & 0 & 0 \\ 0 & 0 & 0 \\ 0 & 0 & 0\end{array}\right]$

The systems of differential equations for each interval of the machine tool lifetime can be solved by transition intensity matrix (21) and reward matrix (23) under initial conditions $V_{i}(0)=0, i=$ $1,2,3$. Thus, the corresponding system of the differential equation for the availability of the machine tool is as follows:

$$
\left\{\begin{array}{c}
\frac{d V_{1}(t)}{d t}=1-\left(\lambda_{F C 1}+\lambda_{F C 2}\right) V_{1}(t)+\lambda_{F C 1} V_{2}(t)+\lambda_{F C 2} V_{3}(t) \\
\frac{d V_{2}(t)}{d t}=\mu V_{1}(t)-\mu V_{2}(t) \\
\frac{d V_{3}(t)}{d t}=\mu V_{1}(t)-\mu V_{3}(t)
\end{array}\right.
$$

After solving the system and finding $V_{i}(t)$, the average availability of the machine tool can be obtained through formula (15).

The cost parameters in this numerical example are shown in Table 1. Meanwhile, it is assumed in this case that the relation between repair cost and repair rate for machine tools expressed in formula (25). Since the higher repair rate means the better repair capacity and correspondingly shorter repair time, which requires more repair resources, like the availability of appropriate maintenance personnel, the required spare parts, maintenance equipment and tools, etc.

$C_{r}^{m}=50 \exp \left(0.053 \cdot \mu^{m}\right)$

where $\mu^{m}$ is the optimal repair rate for the $m$ th interval and $C_{r}^{m}$ is the required repair cost, $m=$ $1,2, \cdots, M$.

Table 1. Cost parameters used in this case

\begin{tabular}{ccc}
$C_{o p}(€ / h r)$ & $C_{p}(€ / h r)$ & $C_{I R}(€)$ \\
\hline 2 & 100 & 20 \\
\hline
\end{tabular}

$C_{o p}$ is the operational cost for this machine tool, $C_{p}$ is the penalty cost of machine tool staying in the failure state and $C_{I R}$ is the average cost of increased rejections.

In the current paper, the lifetime of the machine tool is divided into 20 equal intervals and the instantaneous availability criterion is determined set as $A_{0}(t)=0.99$. Thus, in accordance with the maintenance planning process, the first step in this paper is to determine an optimal repair rates layout for each interval in order to provide $\operatorname{MIN}_{\mu^{m}}\left\{\sum_{m=1}^{M} E\left[R A C_{M T}^{m}\right]\right\}$ and fulfill availability constraint $A(t) \geq 0.99$ during the life cycle.

In order to find the Reliability Associated Cost we should present the reward matrixes $\boldsymbol{r}_{\boldsymbol{R} A \boldsymbol{C}}^{\boldsymbol{m}}$ for 
International Journal of Mathematical, Engineering and Management Sciences

Vol. 4, No. 4, 824-840, 2019

https://dx.doi.org/10.33889/IJMEMS.2019.4.4-065

each interval in the following forms:

$\boldsymbol{r}_{\boldsymbol{R A C}}^{\boldsymbol{m}}=\left[\begin{array}{ccc}C_{o p} & 0 & 0 \\ C_{r}^{m} & C_{p} & 0 \\ C_{r}^{m} & 0 & C_{p}\end{array}\right]$

The system of differential equations (11) for each interval must be solved for transition intensity matrix (21) and reward matrix (26) under initial conditions $V_{i}^{m}((m-1) \tau)=V_{i}^{m-1}((m-$ 1) $\tau), V_{i}^{1}(0)=0, i=1,2,3 ; m=1,2, \cdots, 20$.

The system of differential equations is as follows:

$$
\left\{\begin{array}{c}
\frac{d V_{1}^{m}(t)}{d t}=C_{o p}-\left(\lambda_{F C 1}+\lambda_{F C 2}\right) V_{1}^{m}(t)+\lambda_{F C 1} V_{2}^{m}(t)+\lambda_{F C 2} V_{3}^{m}(t) \\
\frac{d V_{2}^{m}(t)}{d t}=C_{p}+C_{r}^{m} u+\mu V_{1}^{m}(t)-\mu V_{2}^{m}(t) \\
\frac{d V_{3}^{m}(t)}{d t}=C_{p}+C_{r}^{m} u+\mu V_{1}^{m}(t)-\mu V_{3}^{m}(t)
\end{array}\right.
$$

The normal Reliability Associated Cost of the machine tool based on formal (16) can be determined as follows:

$C_{1}=V_{1}^{20}(t)$

Furthermore, in order to find the mean number of FC2 for each interval, the rewards in all matrixes $r_{N}$ are in the following form.

$\boldsymbol{r}_{N}=\left[\begin{array}{lll}0 & 0 & 1 \\ 0 & 0 & 0 \\ 0 & 0 & 0\end{array}\right]$

The systems of differential equations for the mean number of FC2 during each interval of the machine tool lifetime can be solved by transition intensity matrix (21) and reward matrix (29) under initial conditions $V_{N i}^{m}(0)=0, i=1,2,3, m=1,2, \cdots, 20$. Thus, the corresponding system of the differential equation for the mean number of FC2 is as follows:

$$
\left\{\begin{array}{c}
\frac{d V_{N 1}^{m}(t)}{d t}=\lambda_{F C 2}-\left(\lambda_{F C 1}+\lambda_{F C 2}\right) V_{N 1}^{m}(t)+\lambda_{F C 1} V_{N 2}^{m}(t)+\lambda_{F C 2} V_{N 3}^{m}(t) \\
\frac{d V_{N 2}^{m}(t)}{d t}=\mu V_{N 1}^{m}(t)-\mu V_{N 2}^{m}(t) \\
\frac{d V_{N 3}^{m}(t)}{d t}=\mu V_{N 1}^{m}(t)-\mu V_{N 3}^{m}(t)
\end{array}\right.
$$

Therefore, in accordance with the formula (17) and (18), the additional cost incurred by FC2 during the machine tool lifetime obtained as follow:

$C_{2}=\left(C_{I R}+\left(\frac{1}{f}\right) \cdot C_{o p}\right) \cdot \sum_{m=1}^{20} N_{F C 2}^{m}$

The total expected Reliability Associated Cost of the machine tool during lifetime without 
International Journal of Mathematical, Engineering and Management Sciences

Vol. 4, No. 4, 824-840, 2019

https://dx.doi.org/10.33889/IJMEMS.2019.4.4-065

including the overhaul shown as follows:

$\sum_{m=1}^{20} E\left[R A C_{M T}^{m}\right]=E\left[C_{1}\right]+E\left[C_{2}\right]$

The proposed GA implemented by MATLAB to determine the optimal repair rates layout for each interval of the machine tool shown in Table 2, while Table 3 presents the values of Reliability Associated Cost incurred in each interval and cost increment within intervals. Three overhauls should execute during its lifetime, the specific moments and total RAC are as follows:

Table 2. The optimal repair rates layout

\begin{tabular}{cccccccccccc} 
Interval & 1 & 2 & 3 & 4 & 5 & 6 & 7 & 8 & 9 & 10 \\
\hline$\mu / h r$ & 5.3 & 5.26 & 5.2 & 5.2 & 5.2 & 5.18 & 5.25 & 5.23 & 5.21 & 5.19 \\
Interval & 11 & 12 & 13 & 14 & 15 & 16 & 17 & 18 & 19 & 20 \\
$\mu / h r$ & 5.23 & 5.2 & 5.19 & 5.18 & 5.85 & 6.96 & 8.09 & 9.25 & 10.43 & 11.64 \\
\hline
\end{tabular}

Table 3. Cumulative RAC and the cost increment of each interval (€)

\begin{tabular}{ccccccccccc} 
Interval & 1 & 2 & 3 & 4 & 5 & 6 & 7 & 8 & 9 & 10 \\
\hline$R A C^{m}$ & 5454 & 12606 & 21726 & 32979 & 46485 & 62337 & 69865 & 79400 & 91095 & 105062 \\
Increment & -- & 7152 & 9120 & 11253 & 13506 & 15852 & 7528 & 9535 & 11695 & 13967 \\
Interval & 11 & 12 & 13 & 14 & 15 & 16 & 17 & 18 & 19 & 20 \\
$R A C^{m}$ & 116227 & 129640 & 145396 & 161171 & 179415 & 200433 & 224575 & 252232 & 283834 & 319871 \\
Increment & 11165 & 13413 & 15756 & 15775 & 18244 & 21018 & 24142 & 27657 & 31602 & 36037 \\
\hline
\end{tabular}

$\left\{\begin{array}{l}t_{1}=2400 * 6 \mathrm{hrs} \\ t_{2}=2400 * 10 \mathrm{hrs} \\ t_{3}=2400 * 13 \mathrm{hrs}\end{array}\right.$
$E\left[R A C_{M T}\right]=317891+3 * 16000=367871(€)$.

Figure 6 demonstrates the comparisons between the RAC incurred by the corrective maintenance strategy only and that caused by using the maintenance strategy proposed in current paper. 
International Journal of Mathematical, Engineering and Management Sciences

Vol. 4, No. 4, 824-840, 2019

https://dx.doi.org/10.33889/IJMEMS.2019.4.4-065

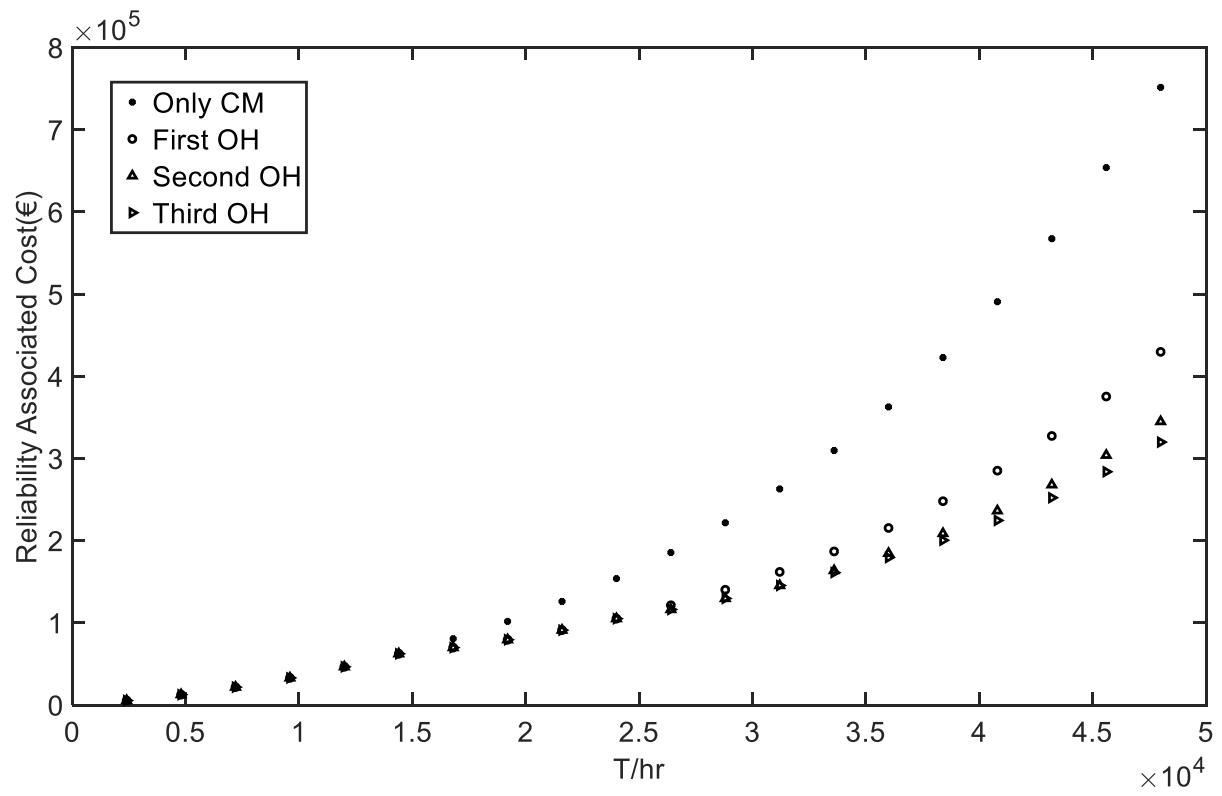

Figure 6. Comparative reliability associated cost between different maintenance strategies

The optimization results and Figure.6 indicate the maintenance strategy proposed in this paper that combine corrective maintenance with overhaul can significantly reduce the Reliability Associated Cost incurred during the life cycle of the machine tool. Compared to the only corrective maintenance strategy, the proposed method can save $51 \%$ of the total RAC.

\section{Conclusions}

A novel Reliability Associated Cost model of machine tools is proposed in the current paper, which takes the impact of shop-floor product quality detection on a machine tool's state into consideration. Thus, the cost incurred by the increased rejections is included in this model, which can better demonstrate the cost caused in the life cycle of machine tools.

This paper studies the maintenance strategy of corrective maintenance combined with the overhaul. It is supposed that the failures occurred before the set overhaul moment are fixed with corrective maintenance and the repair effect of corrective maintenance and overhaul is minimum and imperfect maintenance, respectively. Therefore, the Markov reward approach is implemented to calculate the total Reliability Associated Cost of a machine tool incurred by operation and maintenance activities through its lifetime, and the Genetic Algorithm is the optimal tool for determining the repair rates layout and the overhaul moments. The case study demonstrates that the optimal results can significantly reduce the RAC and achieve the goal of meeting the availability requirement of the machine tool with minimal RAC during its lifetime.

The method in this paper is based on using Markov reward approach and Genetic Algorithm, which are well formalized and suitable for practical applications in reliability engineering for a real-world MSSs reliability measures analysis. Additionally, this method supports engineering decisionmaking. 
International Journal of Mathematical, Engineering and Management Sciences

Vol. 4, No. 4, 824-840, 2019

https://dx.doi.org/10.33889/IJMEMS.2019.4.4-065

\section{Conflict of Interest}

The authors confirm that there is no conflict of interest to declare for this publication.

\section{Acknowledgements}

Currently, the China Scholarship Council (CSC) funds the stay of ZENG Wenbin at the Institute of Energy

Conversion Technology, Munich, Germany.

\section{References}

Atamuradov, V., Medjaher, K., Dersin, P., Lamoureux, B., \& Zerhouni, N. (2017). Prognostics and health management for maintenance practitioners-Review, implementation and tools evaluation. International Journal of Prognostics and Health Management, 8(060), 1-31.

Bolvashenkov, I., Herzog, H.G., Frenkel, I., \& Khvatskin, L. (2018, April). Using Markov reward model for decision making in the choice of optimal type of traction electric motor for icebreaking ship. In 2018 Thirteenth International Conference on Ecological Vehicles and Renewable Energies (EVER) (pp. 1-6). IEEE.

Fakher, H.B., Nourelfath, M. \& Gendreau, M. (2017). A cost minimisation model for joint production and maintenance planning under quality constraints. International Journal of Production Research, 55(8), 114.

Gandhi, K., \& Ng, A.H. (2018, August). Machine maintenance decision support system: a systematic literature review. In Advances in Manufacturing Technology XXXII: Proceedings of the 16th International Conference on Manufacturing Research, incorporating the 33rd National Conference on Manufacturing Research, September 11-13, 2018, University of Skövde, Sweden (Vol. 8, p. 349). IOS Press.

Howard, R. (1960). Dynamic programming and Markov process. Cambridge, Massachusetts: MIT Press.

Kijima, M. (1989). Some results for repairable systems with general repair. Journal of Applied probability, 26(1), 89-102.

Lad, B.K., Shrivastava, D., \& Kulkarni, M.S. (2016). Machine tool reliability. Wiley.

Lisnianski, A., \& Frenkel, I. (2009). Non-homogeneous Markov reward model for aging multi-state system under minimal repair. International Journal of Performability Engineering, 5(4), 303-312.

Lisnianski, A., \& Levitin, G. (2003). Multi-state system reliability: assessment, optimization and applications (Vol. 6). World Scientific Publishing Company.

Lisnianski, A., Ding, Y., Frenkel, I., \& Khvatskin, L. (2007). Maintenance optimization for multi-state aging systems. In Proc. of 5-th International Conference on Mathematical Methods in Reliability, Methodology and Practice, Glasgo, United Kingdom.

Lisnianski, A., Frenkel, I. \& Ding Y. (2010). Multi-state system reliability analysis and optimization for engineers and industrial managers. Springer, London.

Lisnianski, A., Frenkel, I., Khvatskin, L., \& Ding, Y. (2008). Maintenance contract assessment for aging systems. Quality and Reliability Engineering International, 24(5), 519-531.

Mourtzis, D., Vlachou, E., Milas, N., \& Xanthopoulos, N. (2016). A cloud-based approach for maintenance of machine tools and equipment based on shop-floor monitoring. Procedia Cirp, 41, 655-660.

Peysson, F., Mozzati, C., Leon, D., Lafuste, Q., \& Leger, J.B. (2019). Fleet-wide proactive maintenance of machine tools. In Twin-Control (pp. 209-224). Springer, Cham. 
International Journal of Mathematical, Engineering and Management Sciences

Vol. 4, No. 4, 824-840, 2019

https://dx.doi.org/10.33889/IJMEMS.2019.4.4-065

Reason, J. (2000). Cognitive engineering in aviation domain. Lawrence Erlbaum Associates, Mahwah, NJ.

Shagluf, A., Parkinson, S., Longstaff, A.P., \& Fletcher, S. (2018). Adaptive decision support for suggesting a machine tool maintenance strategy: from reactive to preventative. Journal of Quality in Maintenance Engineering, 24(3), 376-399.

Sharma, A., Yadava, G.S., \& Deshmukh, S.G. (2011). A literature review and future perspectives on maintenance optimization. Journal of Quality in Maintenance Engineering, 17(1), 5-25.

Shen, G.X., Zeng, W.B., Zhang, Y.Z., HAN, C.Y. \& LIU, P. (2017). Determination of the average maintenance time of $\mathrm{CNC}$ machine tools based on type II failure correlation. Eksploatacja $i$ Niezawodnosc-Maintenance and Reliability, 19(4): 604-614.

Ungureanu, A.L., Stan, G., \& Butunoi, P.A. (2015). Maintenance strategy optimization for computer numerical control machine tools. In Applied Mechanics and Materials (Vol. 809, pp. 1504-1509). Trans Tech Publications.

Ungureanu, A.L., Stan, G., \& Butunoi, P.A. (2016, August). Reducing maintenance costs in agreement with CNC machine tools reliability. In IOP Conference Series: Materials Science and Engineering (Vol. 145, No. 2, p. 022003). IOP Publishing.

Wan, S., Gao, J., Li, D., Tong, Y., \& He, F. (2015). Web-based process planning for machine tool maintenance and services. Procedia CIRP, 38, 165-170.

Wan, S., Li, D., Gao, J., Roy, R., \& He, F. (2018). A collaborative machine tool maintenance planning system based on content management technologies. The International Journal of Advanced Manufacturing Technology, 94(5-8), 1639-1653.

Wang, M.L., Chen, X.Y., Ding, W.Z., Zhu, H. \& Zhu, L. (2016). Intelligent maintenance of moving joints on large-scale machine tool. Advances in Intelligent Systems Research, 130, 1357-1361.

Xu, W.G. \& Cao, L. (2018). Optimal maintenance control of machine tools for energy efficient manufacturing. The International Journal of Advanced Manufacturing Technology, 1-9. 